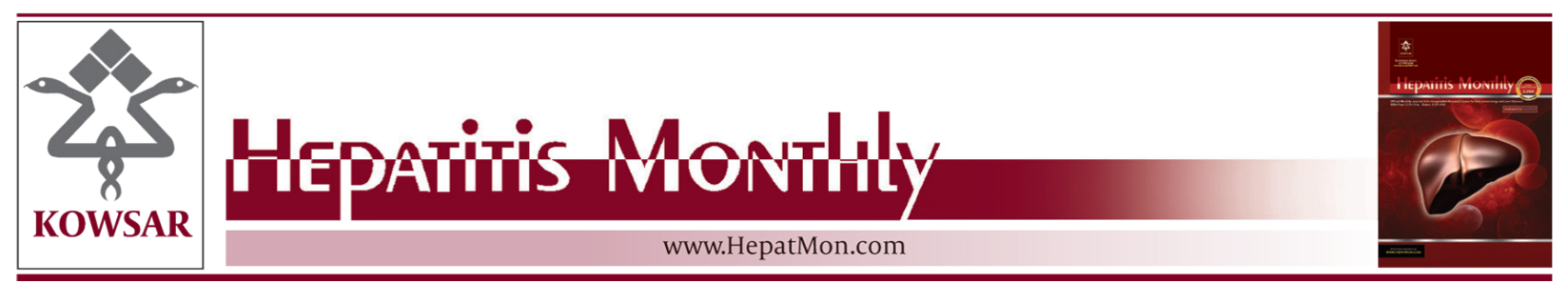

\title{
Multiplex ARMS PCR to Detect 8 Common Mutations of ATP7B Gene in Pa- tients With Wilson Disease
}

\author{
Hassan Dastsooz ${ }^{1,2}$, Mohammad Hadi Imanieh ${ }^{3}$, Seyed Mohsen Dehghani ${ }^{3}$, Mahmood \\ Haghighat ${ }^{3}$, Maryam Moini ${ }^{4}$, Majid Fardaei ${ }^{1,2,5,{ }^{*}}$ \\ ${ }^{1}$ Department of Medical Genetics, Shiraz University of Medical Sciences, Shiraz, IR Iran \\ ${ }^{2}$ Department of Molecular Medicine, Shiraz University of Medical Sciences, Shiraz, IR Iran \\ ${ }^{3}$ Shiraz Transplant Research Center, Gastroenterohepatology Research Center, Namazi Teaching Hospital, Shiraz University of Medical Sciences, Shiraz, \\ IR Iran \\ ${ }^{4}$ Department of Internal Medicine, Gastroenterology and Hepatology Research Center, Shiraz University of Medical Sciences, Shiraz, IR Iran \\ ${ }^{5}$ Stem Cell and Transgenic Technology Research Center, Shiraz University of Medical Sciences, Shiraz, IR Iran \\ *Corresponding author: Majid Fardaei, Department of Medical Genetics, Shiraz University of Medical Sciences, 7134853185, Shiraz, IR Iran. Tel: +98-7112349610, \\ Fax: +98-7112349610, E-mail: mfardaei@sums.ac.ir.
}

\begin{abstract}
A B S T R A C T
Background: Wilson disease is a rare disorder of copper metabolism due to mutation in ATP7B gene. Proper counseling of patients with Wilson disease, and their families necessitates finding mutation in ATP7B gene. Finding mutations in ATP7B gene with 21 exons, and more than 500 mutations is expensive and time-consuming.

Objectives: The aim of this study was to provide a simple multiplex amplification refractory mutation system PCR(M-ARMS-PCR) for screening eight common mutations in ATP7B gene.

Patients and Methods: Two sets of ARMS mutant and normal specific primer pairs were designed for genotyping of p.R778L, p.R969Q, p.H1069Q, and p.3400delC mutations as Set 1 and p.W779G, c.3061-1G > A, p.I1102T, and p.N1270S mutations as Set 2. The Multiplex ARMS assay was then subsequently tested in 65 patients with Wilson disease with known and unknown ATP7B mutations.

Results: Using these two sets, we identified H1069Q mutation in four patients, c.2335T > G mutation in three, c.3061-1G > A splice site mutation in five, c.3305T > C mutation in one, and c.3809A > G mutation in two patients.

Conclusions: The Multiplex ARMS assay used in this study can be an efficient, reliable, and cost effective method as a primary screen for patients with Wilson disease.
\end{abstract}

Keywords: Multiplex Polymerase Chain Reaction; Hepatolenticular Degeneration; Iran

Copyright @ 2013, Kowsar Corp.; Published by Kowsar Corp.

Article type: Research Article; Received: 29 Sep 2012; Revised: 07 Jan 2013; Accepted: 11 Feb 2013; Epub: 16 May 2013

Implication for health policy/practice/research/medical education:

According to some of the recent publications, a couple of predominant mutations have been identified in the ATP7B gene in patients with Wilson disease in Iran. This study will provide a simple multiplex-PCR technique to detect common mutations in ATP7 gene.

-Please cite this paper as:

DastsoozH, ImaniehMH, DehghaniSM, Haghighat M, MoiniM, Fardaei M. Multiplex ARMS PCR to Detect 8 Common Mutations of ATP7B Gene in Wilson Disease Patients. Hepat Mon. 2013;13(5):e8375. DOI: 10.5812/hepatmon.8375

Copyright (C) 2013, Kowsar Corp.; Published by Kowsar Corp.

This is an Open Access article distributed under the terms of the Creative Commons Attribution License (http://creativecommons.org/licenses/by/3.0), which permits unrestricted use, distribution, and reproduction in any medium, provided the original work is properly cited. 


\section{Background}

Wilson disease (WD) (OMIM 277900) is a rare autosomal recessive disorder, characterized by decreased serum ceruloplasmin concentration, increased levels of $24 \mathrm{~h}$ urinary copper excretion, hepatic manifestations, neurological manifestations, and the presence of KF (Kayser-Fleischer) rings (1-5). WD in homozygous state occurs from 1 in 30,000 to 100,000 (2), and it is expected that one in 90 individuals would be heterozygous carrier for this disease (6). Historically, diagnosis is based on the laboratory and clinical findings; however, a molecular approach is required to provide an unambiguous diagnosis of the patients and their families $(7,8)$. WD is caused by different mutations in ATP7B gene (Ref Seq Gene: NG_008806.1) which encodes a copper-transporting p-type 2 ATPase protein $(7,9)$. To date, over 500 mutations have been reported that are distributed across the 21 ATP7B exons, exonintron boundaries, and promoter region. (www.wilsondisease.med.ualberta.ca/search3.asp). Therefore, mutation screening for WD is complicated.

\section{Objectives}

The purpose of this study was to provide a more cost-effective and rapid approach of mutation screening for patients with WD. For this purpose, a multiplex amplification refractory mutation system (M-ARMS) was designed to detect 8 mutations in the ATP7B gene. These mutations were selected on the basis of their frequency in several nations, and also in the south of Iran.

\section{Patients and Methods}

\subsection{Patients and DNA Samples}

Samples consisted of patients with WD referring to Namazi Gastroenterology Center between 2010 and 2012. Diagnosis was made on the basis of an elevated $24 \mathrm{~h}$ urinary copper excretion, or an increased liver copper concentration, low level of serum ceruloplasmin, clinical features such as liver disease, neuropsychiatric disease, and the presence of KF rings. All the patients gave written informed consent before undergoing DNA test for ATP7B mutation analysis according to the ethics committee in Shiraz University of Medical Sciences. Three $\mathrm{ml}$ of the peripheral venous blood samples were collected into EDTA tubes from 65 patients with WD. The blood samples were stored at $-20^{\circ} \mathrm{C}$ until use.DNA was extracted by AccuPrep $₫$ Genomic DNA Extraction Kit (Bioneer, Korea) according to the manufacturer's recommendations. The DNA quantity was measured by NanoDrop (ND1000, USA), and the extracted DNA was stored at $-20^{\circ} \mathrm{C}$ until use.

\subsection{M-ARMS Primer Design}

Two sets of mutant-specific primers (Set 1 and 2) were designed to generate four mutant amplicons, and two control amplicons in each set. Set 1 contained primers for the detection of the most common mutations of ATP7B gene, including c.2333G > T (p.R778L), c.2906G > A (p. R969Q), c.3207C > A
(p.H1069Q), and c.3400delC located in exons 8, 13,14, and 15, respectively, and also two internal control amplicons from the two intronic sequences in ATP7B gene, lower and upper controls (Table 1). Set 2 contained primers for four mutations identified by DHPLC analysis in patients with WD from the south of Iran; some of the mutations were more common (10), including c.2335T > G (p.W779G), c.3061-1G > A, c.3305T $>$ C (p.I1102T), and c.3809A > G (p.N1270S), and two internal controls were the same as Set1 (Table 2). To discriminate hetero- and homozygote variants, two sets of these primer pairs were also designed to create normal and control amplicons, Set $1 A$ and $2 A$. These sets contained primers specific for normal DNA which was different from the Sets 1 and 2 at their 3 ' end nucleotides (Tables 1 and 2). In this case, a heterozygote mutation would be amplified with both normal and mutant primers, but a homozygote mutation would be only amplified with the primer specific for the mutation. These primer pairs were designed, and evaluated on the basis of the ATP7B genomic sequence (GenBank accession no: NG_008806) using several websites and bioinformatics softwares such as Oligocalc, Primer3, Primer-BLAST, NCBI-BLAST, PRIMER Biosoft, and PrimerPlex 2.50. The specificity of the ARMS PCR primers was enhanced by the introduction of an additional mismatch at the second, third, or fourth nucleotide from the 3 ' end of the primer. In the primer in which the 3 ' terminal mismatch was strong (C-C, G-A, and A-A), a weak secondary mismatch was introduced, and if the 3 ' terminal mismatch was weak, a strong secondary mismatch was selected (11). The M-ARMS was standardized on DNA samples with four known mutations of Set 2 (c.2335T > G, c.3061-1G $>\mathrm{A}$, c.3809A $>\mathrm{G}, \mathrm{c} .3305 \mathrm{~T}>\mathrm{C}$ ), and one known mutation of Set 1 (c.3207C > A).The Multiplex ARMS assay was then subsequently tested in 65 patients with Wilson disease with 15 known, and 50 unknown ATP7B mutations.

\subsection{Multiplex ARMS PCR}

The PCR reaction was performed with QIAGEN Multiplex PCR master mix kit (QIAGEN, Germany) using Eppendorf Mastercycler Gradient (Germany). The Multiplex PCR kit contained preoptimized concentrations of HotStar Taq DNA Polymerase, and $\mathrm{MgCl} 2$, plus dNTPs, and a PCR buffer which allowed efficient primer annealing and extension. To begin with, 10x primer mixes (containing each primer at $2 \mu \mathrm{M}$ ) were prepared according to the QIAGEN Multiplex PCR kit. Multiplex PCR reactions were then prepared in 50 1 l containing $25 \mu$ of 2x QIAGEN Multiplex PCR Master Mix, $5 \mu$ of 10x primer mix, 50-100 ng DNA, and enough RNase-free water. Finally, the PCR reaction was performed using Eppendorf Mastercycler Gradient according to the following protocol: $95^{\circ} \mathrm{C}$ for $15 \mathrm{~min}$, then 35 cycles at $94^{\circ} \mathrm{C}$ for 30 s, annealing temperature for $90 \mathrm{~s}\left(\right.$ Set $1: 67^{\circ} \mathrm{C}$, Set $1 \mathrm{~A}: 62^{\circ} \mathrm{C}$, Set $2: 64^{\circ} \mathrm{C}$, Set $2 \mathrm{~A}$ : $65^{\circ} \mathrm{C}$ ), $72^{\circ} \mathrm{C}$ for 45 s followed by a final extension step at $72^{\circ} \mathrm{C}$ for $30 \mathrm{~min}$. Following amplification, $10 \mu \mathrm{l}$ of the PCR reaction was electrophoresed along with a 100-bp ladder (Vivantis) on a $2 \%$ agarose gel and visualized under the UV lightbox (GBOX, SYNGENE, UK). 


\section{Results}

In the present study, the M-ARMS were designed to identify the eight common mutations of ATP7B gene. The ATP7B ARMS assay consisted of two separate PCR sets, and each containing primer for four different mutations. To confirm
PCR success, two pairs of control primers were included in each set. The M-ARMS was standardized on DNA samples with four known mutations of Set 2, and one known mutation of Set 1 . The method was subsequently tested on 67 samples with known and unknown ATP7B gene mutations.

\begin{tabular}{|c|c|c|c|}
\hline Mutation & PCR product $(\mathrm{bp})$ & Exon & Sequences $\left(5^{\prime}>3^{\prime}\right)$ \\
\hline \multirow[t]{3}{*}{$\operatorname{R} 778 \mathrm{~L}(\mathrm{c} .2333 \mathrm{G}>\mathrm{T})$} & 261 (mutant primer) & 8 & F: GCAGCCTTCACTGTCCTTGTCTT \\
\hline & 260 (normal primer) & & R: CTTTGCCAAGTGTTCCAGCCTCA(M) ${ }^{\text {b }}$ \\
\hline & & & R:TTTGCCAAGTGTTCCAGCCTCC $(\mathrm{N})^{\mathrm{b}}$ \\
\hline \multirow[t]{3}{*}{$\operatorname{R969Q}(\mathrm{c} .2906 \mathrm{G}>\mathrm{A})$} & 212 (mutant primer) & 13 & F: CATCTCCCAGACAGAGGTGATCATACA(M) \\
\hline & 209 (normal primer) & & F: CTCCCAGACAGAGGTGATCATCCG (N) \\
\hline & & & R: CAGGATGGGGAAAGCCGTGCTA \\
\hline \multirow[t]{3}{*}{$\operatorname{H1069Q}(\mathbf{c . 3 2 0 7 C}>\mathrm{A})$} & 391 & 14 & F: TGCGGAGGCCAGCAGTGAATAA(M) \\
\hline & (both primers) & & R: TGCGGAGGCCAGCAGTGAATAC (N) \\
\hline & & & R: TGTCAAAGCACTGAGTTTCCAGACTG \\
\hline \multirow[t]{3}{*}{ c.3400delC } & 492 (mutant primer) & 15 & F: TCCTTTCCAGTCGGTAACCTGTTCA \\
\hline & 491 (normal primer) & & R: AGCCAGCAATACCTTTTTCTGCGTA (M) \\
\hline & & & R: CCAGCAATACCTTTTTCTGCGGGAA(N) \\
\hline \multirow[t]{2}{*}{ Lower control } & 153 & & F: AGTGGTCGTTTTAGCAGCAACAGAG \\
\hline & & & R: GTGTTCATGTTACTGGGCCATCTCC \\
\hline \multirow[t]{2}{*}{ Upper control } & 585 & & F: CCACCGTCAGAGGAAGGAGAATTTC \\
\hline & & & R: CTAGGTCAATGAAGAAGACCCTGTACAC \\
\hline
\end{tabular}

\footnotetext{
${ }^{a}$ Mutant-specific nucleotide is shown in bold and italic letter. Deliberate mismatch is underlined

${ }^{b} \mathrm{M}$, mutant-specific primers in Set 1; N, normal-specific primers in Set $1 \mathrm{~A}$
}

\begin{tabular}{|c|c|c|c|}
\hline Mutation & PCR product (bp) & Exon & Sequences $\left(5^{\prime}>3^{\prime}\right)$ \\
\hline \multirow[t]{3}{*}{ W779G $($ c.2335T > G) } & 294 (both primers) & 8 & F: TCGCTCATTGAACTCTCCTCCCT \\
\hline & & & R:ACCTTTGCCAAGTGTTCCAGACC(M) \\
\hline & & & R: ACCTTTGCCAAGTGTTCCAGTCA $(\mathrm{N})^{\mathrm{b}}$ \\
\hline \multirow[t]{3}{*}{ I1102T $(c .3305 T>C)$} & 415 (both primers) & 15 & F: GCAGTGCCAGGCTGTGCAAC(M) \\
\hline & & & F: GCAGTGCCAGGCTGTGCAAT (N) \\
\hline & & & R: CTCTGTAGCTTATGAGAAGCAAGACCG \\
\hline \multirow[t]{3}{*}{ c.3061-1G >A } & 330 (both primers) & 14 & F: CAGTGAGTTGTGGTTGTTTTTGCCAA(M) \\
\hline & & & F: CAGTGAGTTGTGGTTGTTTTTGCCAG (N) \\
\hline & & & R: CTCTAAGTGGTTTTCCAGACCACACAG \\
\hline \multirow[t]{3}{*}{$\operatorname{N1270S}($ c.3809A $>G)$} & 507 (both primers) & 18 & F: CCATGGTGGGGGATGGGGTAAG(M) \\
\hline & & & F: CCATGGTGGGGGATGGGGTAAA (N) \\
\hline & & & R: GTTTCAGGTCCTCTCCACAGTTTCTC \\
\hline \multirow[t]{2}{*}{ Lower control } & 153 & & F: AGTGGTCGTTTTAGCAGCAACAGAG \\
\hline & & & R: GTGTTCATGTTACTGGGCCATCTCC \\
\hline \multirow[t]{2}{*}{ Upper control } & 585 & & F: CCACCGTCAGAGGAAGGAGAATTTC \\
\hline & & & R:CTAGGTCAATGAAGAAGACCCTGTACAC \\
\hline
\end{tabular}

\footnotetext{
${ }^{\mathrm{a}}$ Mutant-specific nucleotide is shown in bold and italic letter. Deliberate mismatch is underlined

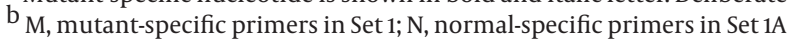




\subsection{Set 1 and Set $1 A$ of Multiplex PCR}

Using M-ARMS for Set 1, c.3207C>A, mutation was detected in four of 65 patients with WD (cases 2, 4, 11, and 18) (Figure 1-A). Presence of this mutation was confirmed by direct DNA sequencing. Set $1 \mathrm{~A}$ was also performed for patients with the compound heterozygote and homozygote c.3207C > A mutation to confirm the efficiency of this method for genotyping (Figure 1-B). In case 18 with the heterozygote c.3207C > A mutation, Set 1 of M-ARMS PCR showed only the control bands (153 bp, and $585 \mathrm{bp}$ ), and the related mutant band (391 bp), but Set $1 \mathrm{~A}$ showed all bands expected to be detected in the presence of normal allele (Figure 1-B, third and fourth wells). Patient with the homozygote c.3207C > A mutation (case 4) showed the control bands (153 bp, and $585 \mathrm{bp}$ ), and the related mutant band ( $391 \mathrm{bp}$ ) with Set 1 , and all bands except the band for this mutation with Set $1 \mathrm{~A}$ (Figure $1-B$, fifth and sixth wells). Normal DNA showed only control amplicons using Set 1 (Figure 1-B, first well), but all normal amplicons were amplified using Set $1 \mathrm{~A}$ (Figure 1-B, second well). Sequencing graph for this mutation was shown in figure 1 -C. These observations confirmed that case 18 was a compound heterozygote.

Figure 1. Chromatograms and Gel Electrophoresis Images of the Mutation, and the DNA Bands of Set 1 and $1 \mathrm{~A}$

(A)

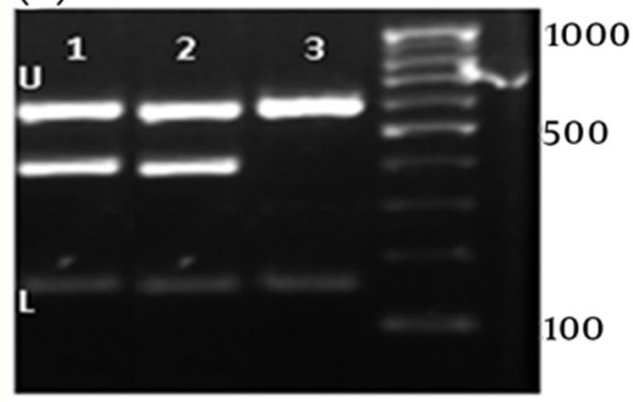

(B)

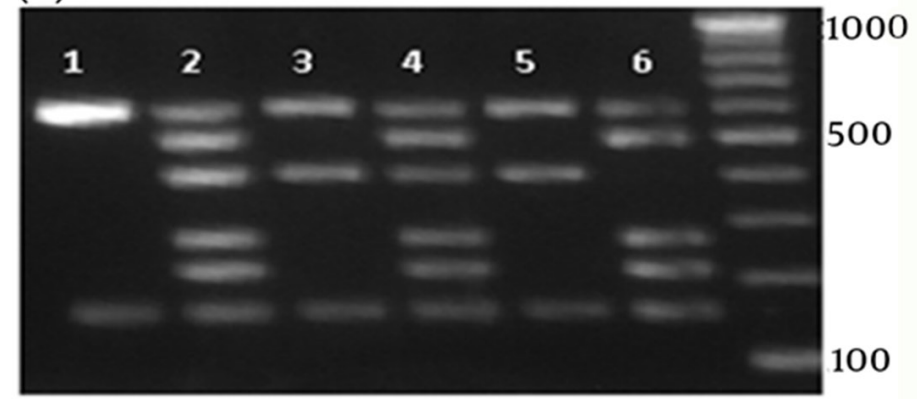

(C)

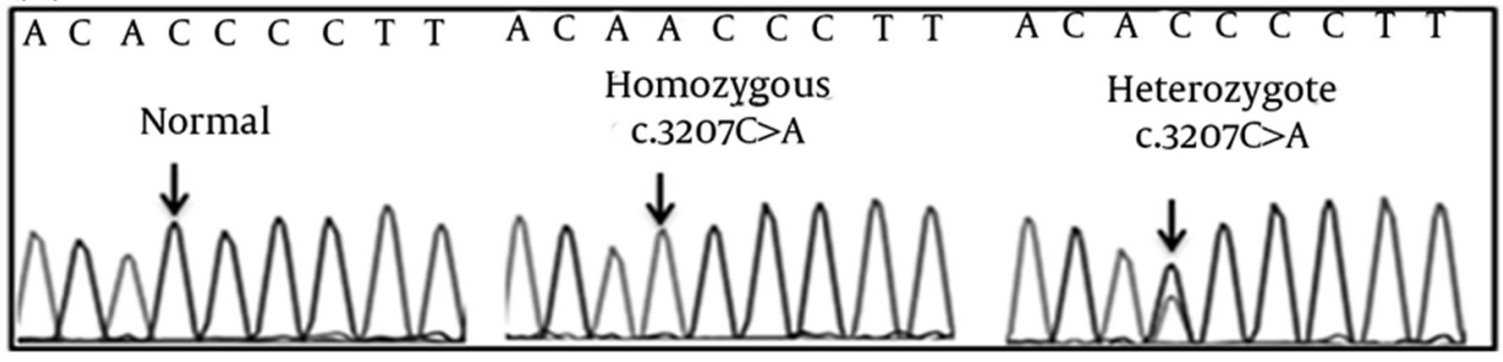

(A) Gel electrophoresis of Set 1, U: upper and L: lower controls are depicted for all lines. 1 and 2 show the band for the c.3207C > A mutation.3: Normal DNA which shows only the control amplicons. 100bp DNA ladder is depicted in left. (B) Gel electrophoresis of the DNA bands of the hetero- and homozygote c.3207C > A mutation. 1 and 2 shows the normal control DNA using Set 2 and 2A, respectively. 3 and 4 show the heterozygote c.3207C $>$ A mutation. 5 and 6 show patients with the homozygote c.3207C > A mutation. 10obp DNA ladder is depicted in left. (C) Chromatograms of main mutation detected in Set 1.

\subsection{Set 2 and $2 A$ of Multiplex PCR}

M-ARMS PCR of Set 2 was performed for 65 patients with Wilson disease. Similarly for Set 2, all samples showed both control bands, and bands from c.2335T > G mutation in three cases, c.3061-1G > A splice site mutation in four cases, c.3305T > C mutation in one case, and c.3809A $>\mathrm{G}$ mutation in two cases (examples of cases with these mutations are shown in Figure 2-A. first, 2nd, 5th, and 7th wells). Distribution of ATP7B mutations $(n=65)$ detected by Set 1 and 2 of multiplex ARMS, and sequencing technique are given in Table 3. No band other than the internal control was amplified in the DNA without these mutations (Figure 2-A. 3rd, 4th, and 6th wells). The assay was designated as a fail try if both PCR control bands were not amplified, and the M-ARMS assay was repeated. To confirm the efficiency of this method for genotyping, Set $2 \mathrm{~A}$ was performed for c.2335T $>$ G mutation in samples with a homozygote and a heterozygote (a sample from mother of this homozygous patient) condition. Set $2 \mathrm{~A}$ was also performed for c.3061-1G > A mutation in patients with a homozygote, and a compound heterozygote condition. Using Set 2 of M-ARMS assay, patients with the homozygote c.2335T > G mutation (case 3), and c.3061-1G > A mutation (case 8 ) showed the control bands (153 bp, and 585 bp), and the related mutant bands (294 bp for c.2335T > G and 330 bp for c.3061-1G > A), and using Set 2A all bands except the bands for these mutations were amplified (Figure $2-B, 8$ th, and 9 th wells for the case 3 ; and 6 th and 7 th wells for case 8). 


\begin{tabular}{|c|c|c|c|c|c|c|c|}
\hline \multicolumn{3}{|c|}{ Genotype } & \multicolumn{4}{|c|}{ M. ARMS in Set 1} & \multirow[t]{2}{*}{ Patient } \\
\hline Sequencing & C. $\mathrm{H}^{\mathrm{a}}$ & $\mathbf{H}^{\mathrm{a}}$ & c.340odelC Exon 15 & c.3207C > A Exon 14 & c.2906G > AExon 13 & c.2333G $>T^{4}$ Exon 8 & \\
\hline c. $3207 \mathrm{C}>\mathrm{A}$ & - & + & - & + & - & & Case 2 \\
\hline c.3207C $>$ A & - & + & - & + & - & - & Case 4 \\
\hline c.3207C $>$ A & - & + & - & + & - & - & Case 11 \\
\hline c.3207C $>$ A & + & - & - & + & - & - & Case 18 \\
\hline \multicolumn{3}{|c|}{ Genotype } & \multicolumn{4}{|c|}{ M. ARMS in Set 2} & Patient \\
\hline Sequencing & C. H & $\mathbf{H}$ & c.3809A > GExon18 & C.3305T >CExon15 & c.3061-1G >AExon14 & c.2335T > GExon8 & \\
\hline c.3809A $>$ G & - & + & + & - & - & - & Case 1 \\
\hline c.2335T $>$ G & - & + & - & - & - & + & Case 3 \\
\hline c. $2335 \mathrm{~T}>\mathrm{G}$ & - & + & - & - & - & + & Case 5 \\
\hline c.3061-1G $>$ A & - & + & - & - & + & - & Case 6 \\
\hline c.3061-1G $>$ A & - & + & - & - & + & - & Case 7 \\
\hline c.3061-1G $>$ A & - & + & - & - & + & - & Case 8 \\
\hline C. $3305 \mathrm{~T}>\mathrm{C}$ & - & + & - & + & - & - & Case 10 \\
\hline c. $2335 \mathrm{~T}>\mathrm{G}$ & - & + & - & - & - & + & Case 12 \\
\hline c.3809A $>$ G & - & + & + & - & - & - & Case 14 \\
\hline c.3061-1G $>$ A & - & + & - & - & + & - & Case 15 \\
\hline c.3061-1G $>$ A & + & - & - & - & + & - & Case 17 \\
\hline
\end{tabular}

${ }^{\mathrm{a}} \mathrm{H}$, homozygote, C.H, compound heterozygote

Figure 2. Chromatograms and gel Electrophoresis Images of the Mutation and the DNA Bands of Set 2 and 2A

A)

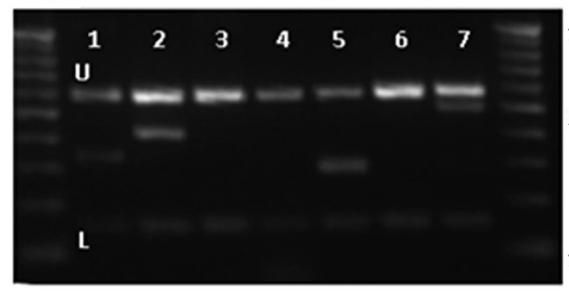

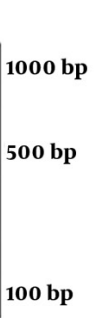

B)

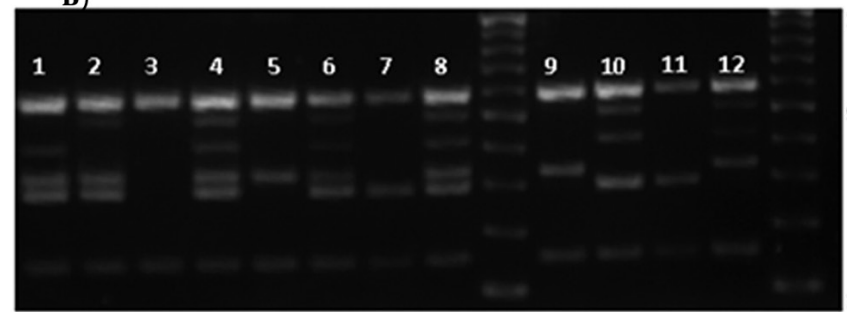

$100 \mathrm{bp}$

C)

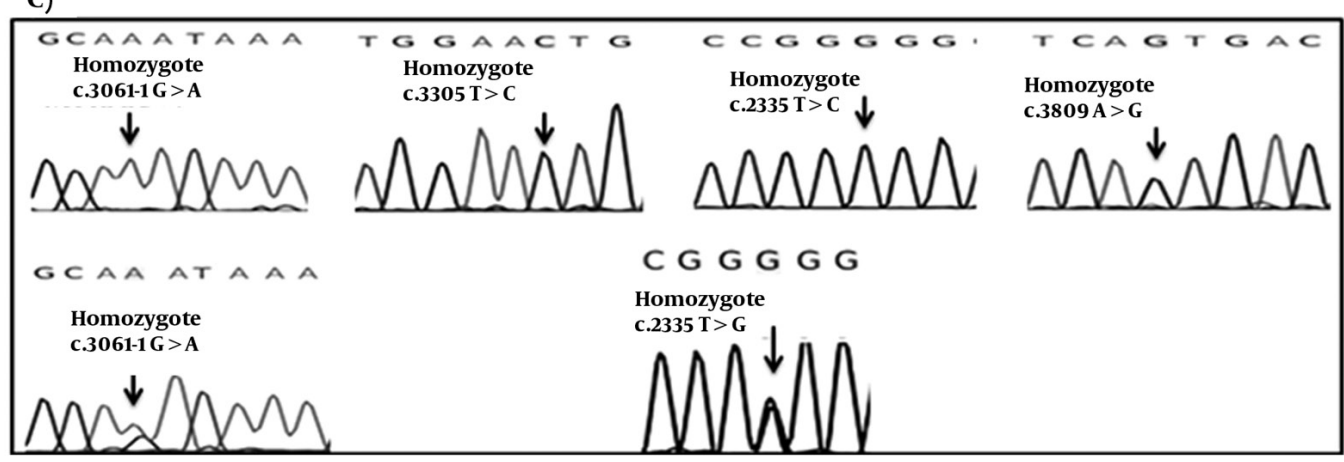

(A) Gel electrophoresis of Set1,U: upper and L: lower controls are depicted for all lines.3rd, 4th, and 6th wells show bands for the normal DNA.100bp DNA ladder is depicted in right and left. Using Mix Set2, Samples in 1st, 2nd, 5th, and 7th wells show the band for c.3061-1G $>$ A, c.3305T $>$ C, c.2335T $>$ G, and c.3809A $>$ G mutations, respectively. (B) Gel electrophoresis of the DNA bands of the hetero-and homozygote c.2335T $>\mathrm{G}$, and c.3061-1G $>$ A mutations. 1: the normal DNA with Set 2A.2 and 3: heterozygotec.3061-1G $>$ A mutation. 4 and 5: heterozygote c.2335T> G mutation;6and 7:homozygotec.3061-1G > A mutation.8and 9:homozygotec.2335T>G.100bp DNAladder is depicted in middle and left.(C)Chromatograms of main mutation detected in Set1. 
In the mother of case 3 with the heterozygote c.2335T > G mutation, Set 2 of M-ARMS PCR showed only the control bands (153 bp, and 585 bp), and the mutant band (294 bp), but Set $2 \mathrm{~A}$ showed all bands expected to be detected in the presence of the normal allele (Figure 2-B, 4th and 5th wells). In case 17 with the heterozygote c.3061-1G $>$ A splice site mutation, Set 2 of M-ARMSPCR showed only the control bands (153 bp, and $585 \mathrm{bp}$ ), and mutant band (330 bp), but Set $2 \mathrm{~A}$ showed all bands expected to be detected in the presence of the normal allele (Figure 2-B, 2nd and 3rd wells). Normal DNA showed all normal amplicons using Set 2A (Figure 2-B, first well); these observations confirmed that case 17 , and the mother of case 3 had the heterozygote mutation. Sequencing graph for these mutations were shown in figure 2-C.

\section{Discussion}

Several mutations of ATP7B gene have been reported with high frequency in different nations. The common mutations of ATP7B gene in patients with WD originating from Eastern, Northern, and Central European are H1069Q (at exon 14) (12-15), R969Q (at exon 13) (12-15), and 3400delC (at exon15) $(12,13,15,16)$. R778L (at exon8) is the most common mutation in the East Asian population $(17,18)$ (database maintained at the University of Alberta: www.wilsondisease.med.ualberta.ca/search3.asp). Mutation used for these two sets were also detected in Iranian population (19). These observations suggest that exons 8 , 13,14 , and 15 may be four hot spots for the identification of ATP7B mutations in several nations. In specific populations with high frequencies of the most common mutations, mutation screening approaches which detect such mutations have been described. To investigate several common mutations, M-ARMS PCR can be very useful and cost-effective. The purpose of this study was to determine the efficiency of an M-ARMS assay for the identification of the most common ATP7B gene mutations identified in many populations. To have successful results of ARMS assay, the melting and annealing temperature of all primers in each set was very similar, and also all primer pairs had similar concentrations in the final mix. Overall, in this study, the M-ARMS PCR detected 5 different mutations in 15 cases. No false-negative or false-positive results were obtained using this M-ARMS assay. Exact identification of the mutations in the samples using ARMS assay confirmed the high accuracy of this assay. To detect common mutations of ATP7B mutations in the south of Iran, a procedure such as the M-ARMS is efficient and practical, because several ATP7B mutations can be simultaneously screened. ARMS procedure which detects several mutations of a given gene in different primer Multiplexes is available and used in clinical diagnoses (20-22). Therefore it would be possible to increase the detection of ATP7B gene mutations in the south of Iran by adding additional appropriate primer Multiplexes. The M-ARMS protocol described in this study has provided an accurate, rapid, inexpensive, and direct molecular testing for WD. Depending upon current and future studies, it would be possible to optimize and establish the M-ARMS-PCR for other common mutations of ATP7B for the Southern Iranian population.

\section{Acknowledgements}

The present article was extracted from the thesis written by Hassan Dastsooz. The authors declare that they have no competing interests.

\section{Financial Disclosure}

We have no financial interests related to the material in the manuscript.

\section{Authors' Contribution}

Majid Fardaei designed the project, contributed to the interpretation of the data, and the revision of the manuscript. Hassan Dastsooz performed the experiments, contributed to the interpretation of the data, and the revision of the manuscript. Dr Mohammad Hadi Imanieh, Dr. Mahmood Haghighat, Dr. Seyed Mohsen Dehghani, and Dr. Maryam Moini diagnosed and critically followed-up the patients.

\section{Funding/Support}

This project was financially supported by Shiraz University of Medical Sciences grant No.5615.

\section{References}

1. Cauza E, Maier-Dobersberger T, Polli C, Kaserer K, Kramer L, Ferenci P. Screening for Wilson's disease in patients with liver diseases by serum ceruloplasmin. J Hepatol. 1997;27(2):358-62.

2. Compston A. Progressive lenticular degeneration: a familial nervous disease associated with cirrhosis of the liver, by S. A. Kinnier Wilson, (From the National Hospital, and the Laboratory of the National Hospital, Queen Square, London) Brain 1912: 34; 295 509. Brain. 2009;132(Pt 8):1997-2001.

3. Frommer D, Morris J, Sherlock S, Abrams J, Newman S. KayserFleischer-like rings in patients without Wilson's disease. Gastroenterology. 1977;72(6):1331-5.

4. Merle U, Schaefer M, Ferenci P, Stremmel W. Clinical presentation, diagnosis and long-term outcome of Wilson's disease: a cohort study. Gut. 2007;56(1):115-20

5. Steindl P, Ferenci P, Dienes HP, Grimm G, Pabinger I, Madl C, et al Wilson's disease in patients presenting with liver disease: a diagnostic challenge. Gastroenterology. 1997;113(1):212-8.

6. Scheinberg IH, Sternlieb I, Walshe JM. Wilson's disease and Kayser-Fleischer rings. Ann Neurol. 1986;19(6):613-4.

7. Ferenci P. Regional distribution of mutations of the ATP7B gene in patients with Wilson disease: impact on genetic testing. Hum Genet. 2006;120(2):151-9.

8. Roberts EA, Schilsky ML. Diagnosis and treatment of Wilson disease: an update. Hepatology. 2008;47(6):2089-111.

9. Petrukhin K, Lutsenko S, Chernov I, Ross BM, Kaplan JH, Gilliam TC. Characterization of the Wilson disease gene encoding a Ptype copper transporting ATPase: genomic organization, alternative splicing, and structure/function predictions. Hum Mol Genet. 1994;3(9):1647-56.

10. Dastsooz H, Dehghani SM, Imanieh MH, Haghighat M, Moini M, 
Fardaei M. A new ATP7B gene mutation with severe condition in two unrelated Iranian families with Wilson disease. Gene. 2013;514(1):48-53.

11. Kwok S, Kellogg DE, McKinney N, Spasic D, Goda L, Levenson C, et al. Effects of primer-template mismatches on the polymerase chain reaction: human immunodeficiency virus type 1 model studies. Nucleic Acids Res. 1990;18(4):999-1005.

12. Caca K, Ferenci P, Kuhn HJ, Polli C, Willgerodt H, Kunath B, et al. High prevalence of the H1069Q mutation in East German patients with Wilson disease: rapid detection of mutations by limited sequencing and phenotype-genotype analysis. J Hepatology. 2001;35(5):575-81.

13. Firneisz G, Lakatos PL, Szalay F, Polli C, Glant TT, Ferenci P. Common mutations of ATP7B in Wilson disease patients from Hungary. Am J Med Genet. 2002;108(1):23-8.

14. Loudianos G, Dessi V, Lovicu M, Angius A, Altuntas B, Giacchino $\mathrm{R}$, et al. Mutation analysis in patients of Mediterranean descent with Wilson disease: identification of 19 novel mutations. J Med Genet. 1999;36(11):833-6.

15. Panagiotakaki E, Tzetis M, Manolaki N, Loudianos G, Papatheodorou A, Manesis E, et al. Genotype-phenotype correlations for a wide spectrum of mutations in the Wilson disease gene (ATP7B). Am J Med Genet A. 2004;131(2):168-73.

16. Deguti MM, Genschel J, Cancado EL, Barbosa ER, Bochow B, Mucenic $\mathrm{M}$, et al. Wilson disease: novel mutations in the ATP7B gene and clinical correlation in Brazilian patients. Hum Mutat. 2004;23(4):398.

17. Kim EK, Yoo OJ, Song KY, Yoo HW, Choi SY, Cho SW, et al. Identification of three novel mutations and a high frequency of the Arg778Leu mutation in Korean patients with Wilson disease. Hum Mutat. 1998;11(4):275-8.

18. Shimizu N, Nakazono H, Takeshita Y, Ikeda C, Fujii H, Watanabe A, et al. Molecular analysis and diagnosis in Japanese patients with Wilson's disease. Pediatrics International. 1999;41(4):409-13.

19. Zali N, Mohebbi SR, Esteghamat S, Chiani M, Haghighi MM, Hosseini-Asl SM, et al. Prevalence of ATP7B Gene Mutations in Iranian Patients With Wilson Disease. Hepat Mon. 2011;11(11):890-4.

20. Lacerra G, Musollino G, Di Noce F, Prezioso R, Carestia C. Genotyping for known Mediterranean alpha-thalassemia point mutations using a multiplex amplification refractory mutation system. Haematologica. 2007;92(2):254-5.

21. Piccioli P, Serra M, Gismondi V, Pedemonte S, Loiacono F, Lastraioli S, et al. Multiplex tetra-primer amplification refractory mutation system PCR to detect 6 common germline mutations of the MUTYH gene associated with polyposis and colorectal cancer. Clin Chem. 2006;52(4):739-43.

22. Taylor A, Tabrah S, Wang D, Sozen M, Duxbury N, Whittall R, et al. Multiplex ARMS analysis to detect 13 common mutations in familial hypercholesterolaemia. Clin Genet. 2007;71(6):561-8. 